\title{
Can viscous fiber lower glycemic markers in type 2 diabetes?
}

\section{The first meta-analysis to focus on viscous dietary fiber in T2D suggests a potential role for this supplement in improving glycemic control.}

\section{PRACTICE CHANGER}

Unless contraindicated, recommend viscous fiber supplementation to your patients with type 2 diabetes (T2D), in addition to the usual evidence-based standards of care, to improve markers of glycemic control.

\section{STRENGTH OF RECOMMENDATION}

C: Based on a meta-analysis and systematic review of 28 randomized controlled trials, without discussion of patient-oriented outcomes.

Jovanovski E, Khayyat R, Zurbau A, et al. Should viscous fiber supplements be considered in diabetes control? Results from a systematic review and meta-analysis of randomized controlled trials. Diabetes Care. 2019;42:755-766. Published correction appears in Diabetes Care 2019;42:1604.

\section{ILLUSTRATIVE CASE}

A 57-year-old man who was given a diagnosis of T2D a year ago presents for an office visit. His hemoglobin $\mathrm{A} 1 \mathrm{C}$ level at diagnosis was $8.3 \%$. He is otherwise healthy and has been adhering well to a plan of metformin $1000 \mathrm{mg}$ twice daily, regular exercise, and a low-carbohydrate diet you recommended. His most recent hemoglobin $\mathrm{A} 1 \mathrm{C}$ is $7.3 \%$. He is pleased with his progress, so he is discouraged when you tell him that he is not yet at goal. He asks if there are other things that he can do to further lower his hemoglobin A1C. What can you recommend for him?

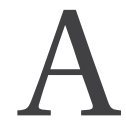
ccording to the National Diabetes Statistics Report, 2020 from the Centers for Disease Control and Preven- tion, approximately 34.1 million US adults $\geq 18$ years of age (13\% of the adult population) have diabetes, $50 \%$ of whom have a hemoglobin A1C > 7\%. The report also states that approximately 88 million US adultsmore than one-third of the population-have prediabetes. $^{2}$

The American Diabetes Association (ADA) estimated that diabetes-related health care costs in the United States for 2017 totaled $\$ 237$ billion, an increase of $26 \%$ from 2012. More than $\$ 30$ billion of this expense comes directly from diabetes medications; the remainder of these costs are related to lost wages, clinic visits, hospitalizations, and treatment for diabetic complications and comorbidities. After controlling for age and gender, medical expenditures for people with diabetes are 2.3 times higher than for those without diabetes. ${ }^{3}$

The 2019 ADA Nutrition Therapy for Adults With Diabetes or Prediabetes: A Consensus Report makes general recommendations concerning fiber intake for patients with diabetes or prediabetes, stating that these patients should consume approximately $14 \mathrm{~g}$ of fiber for every $1000 \mathrm{kcal}$ consumed, giving preference to whole-food sources rather than supplements. ${ }^{4}$ The report indicates that some studies have shown hemoglobin AlC reductions of $0.2 \%$ to $0.3 \%$ with daily fiber intake exceeding $50 \mathrm{~g}$. However, this level of intake can cause unpleasant gastrointestinal adverse effects, including bloating, diarrhea, and flatulence. ${ }^{4,5}$
Erica S. Meisenheimer, MD, MA; Bob Marshall, MD, MPH, MISM, FAAFP, FAMIA; Samuel M.

Tiglao, DO, FAAFP; Tyler S. Rogers, MD; David C. Bury, DO, FAAFP; Michael M. Dickman, DO, FAAFP; Robert C. Oh, MD, MPH, FAAFP

Family Medicine Residency, Madigan Army Medical Center, Joint Base LewisMcChord, WA

DEPUTY EDITOR Jennie B. Jarrett, PharmD, BCPS, MMedEd, FCCP

Family Medicine Residency, Madigan Army Medical Center, Joint Base LewisMcChord, WA

doi: 10.12788/jfp.0141 


\section{$>$}

As an adjunct to standard of care, viscous fiber supplements significantly improved hemoglobin A1C and other glycemic markers in patients with T2D.

\section{STUDY SUMMARY}

\section{Effect on A1C exceeded the FDA threshold for new drugs}

This systematic review and meta-analysis searched MEDLINE, Embase, and the Cochrane Central Register of Controlled Trials to identify randomized controlled trials that studied the effects of viscous fiber supplementation on glycemic control in patients with T2D. Eligible studies included those that: (1) had a duration $\geq 3$ weeks; (2) allowed isolation of the viscous fiber effects; and (3) reported at least 1 of the following glycemic measures: hemoglobin A1C ( $\mathrm{n}=1148$ patients), fasting glucose $(\mathrm{n}=1394)$, fasting insulin ( $\mathrm{n}=228)$, homeostatic model assessment of insulin resistance (HOMA-IR; $\mathrm{n}=652)$, and fructosamine $(\mathrm{n}=23)$.

Data were pooled using the generic inverse variance method and expressed as mean difference (MD) with $95 \%$ confidence intervals (CIs). Heterogeneity was assessed and quantified (Cochran $Q$ and $I^{2}$ statistics, respectively). $I^{2} \geq 50 \%$ indicates substantial heterogeneity. The Grading of Recommendations Assessment, Development, and Evaluation (GRADE) approach was used to evaluate the overall strength of evidence.

Twenty-eight eligible studies were compared. The median age of included patients was 60 years. The median dose of viscous fiber was $13.1 \mathrm{~g} / \mathrm{d}$ (range, 2.55-21). Viscous fiber type varied between the studies and included psyllium, guar gum, $\beta$-glucan, and konjac, and was consumed in powder, tablet, capsule, and limited food-based forms (in 1 of the included studies). The median trial duration was 8 weeks, with 11 trials lasting $\geq 12$ weeks.

The study found moderate-grade evidence that, when added to standard of care, viscous fiber supplementation reduced hemoglobin AlC (MD $=-0.58 \%$; 95\% CI, -0.88 to $\left.-0.28 ; P=.0002 ; I^{2}=91 \%\right)$, fasting glucose $(\mathrm{MD}=-14.8 \mathrm{mg} / \mathrm{dL} ; 95 \% \mathrm{CI}$, -23.8 to $\left.-5.58 ; P=.001 ; I^{2}=92 \%\right)$, and HOMA-IR (MD $=-1.89 ; 95 \% \mathrm{CI},-3.45$ to -0.33 ; $\left.P=.02 ; I^{2}=94 \%\right)$ compared with control. The effect on hemoglobin AlC exceeds the $\geq 0.3 \%$ threshold established by the US Food and Drug Administration for new antihyperglycemic drug development. There was no significant effect on fasting insulin or fructosamine, although the sample size for fructosamine was small. No significant evidence of a dose-dependent response effect was found. The studies had substantial heterogeneity. No evaluation of potential or real harm was noted in the analysis.

\section{WHAT'S NEW}

\section{Potential glycemic benefit without large dietary increase}

The glucose-lowering effects of increased fiber intake have often been hypothesized, but this meta-analysis is the first to focus specifically on the effect of viscous fiber supplements in patients with T2D. Prior meta-analyses, including those cited in the 2019 ADA recommendations mentioned above, included primarily whole-food dietary sources of fiber in the treatment arms and generally had more modest effects on

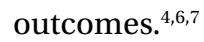

By focusing on viscous fiber supplements, this study isolated the effect of these supplements vs fiber-rich dietary changes. It illustrates a greater potential benefit with supplements than whole-food dietary sources of fiber, and at a lower dose of fiber than was seen in prior studies without requiring substantial increases in caloric intake. Viscous fiber supplementation is a potential adjunct to the usual evidence-based standards of care for glycemic control in patients with T2D.

\section{CAVEATS}

\section{Limited study durations may raise uncertainty about long-term benefits}

This meta-analysis does have its limitations. The heterogeneity among the studies analyzed makes it difficult to establish a single recommendation regarding dose, type, and brand of fiber to be used. Only 11 of the 28 studies lasted longer than 12 weeks, with a median duration of 8 weeks, making any long-term effects on hemoglobin AlC unknown. No adverse effects or reactions were described to evaluate safety and tolerability of the viscous fiber supplementation. No patient-oriented outcomes were reported. 


\section{CHALLENGES TO IMPLEMENTATION}

\section{Patients may not be eager}

\section{to supplement with viscous fiber}

The biggest challenge to implementation is patient compliance. Some forms of supplemental fiber are less palatable than others and may cause unpleasant gastrointestinal adverse effects, which may be an impediment for some patients. Cost may also be an issue for some patients. Diabetes medications can be expensive; however, they are often covered, at least partially, by medical insurance. Over-the-counter supplements are unlikely to be covered for most patients.

JFP

\section{ACKNOWLEDGEMENT}

The PURLs Surveillance System was supported in part by Grant Number UL1RR024999 from the National Center for Research Resources, a Clinical Translational Science Award to the University of Chicago. The content is solely the responsibility of the authors and does not necessarily represent the official views of the National Center for Research Resources or the National Institutes of Health.
Copyright $\odot$ 2021. The Family Physicians Inquiries Network. All rights reserved.

References

1. Jovanovski E, Khayyat R, Zurbau A, et al. Should viscous fiber supplements be considered in diabetes control? Results from a systematic review and meta-analysis of randomized controlled trials. Diabetes Care. 2019;42:755-766. Published correction appears in Diabetes Care. 2019;42:1604.

2. CDC. National Diabetes Statistics Report, 2020. Estimates of Diabetes and Its Burden in the United States. Atlanta, GA: Centers for Disease Control and Prevention, US Dept of Health and Human Services; 2020.

3. American Diabetes Association. Economic costs of diabetes in the U.S. in 2017. Diabetes Care. 2018;41:917-928.

4. Evert AB, Dennison M, Gardner CD, et al. Nutrition therapy for adults with diabetes or prediabetes: a consensus report. Diabetes Care. 2019;42:731-754.

5. American Diabetes Association. 5. Lifestyle management: Standards of Medical Care in Diabetes-2019. Diabetes Care. 2019;42(suppl 1):S46-S60.

6. Post RE, Mainous AG III, King DE, et al. Dietary fiber for the treatment of type 2 diabetes mellitus: a meta-analysis. J Am Board Fam Med. 2012;25:16-23.

7. Jenkins DJA, Kendall CWC, Augustin LSA, et al. Effect of legumes as part of a low glycemic index diet on glycemic control and cardiovascular risk factors in type 2 diabetes mellitus: a randomized controlled trial. Arch Intern Med. 2012;172:1653-1660.

\section{. MEDJOBNETWORK, com Physician • NP/PA Career Center}

\section{The first mobile job board for Physicians, NPs, and PAs}

Mobile Job Searches-access MedJobNetwork.com on the go from your smartphone or tablet

Advanced Search Capabilities - search for jobs by specialty, job title, geographic location, employer, and more

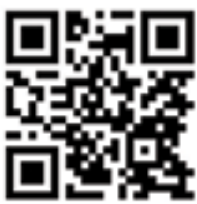

Scan this QR code to access the mobile version of MedJobNetwork.com 